\title{
EDUCAÇÃO INFANTIL: A ORIGEM DOS ALIMENTOS
}

\author{
Sarah da Silva Pereira ${ }^{1}$ \\ Paloma Rosa dos Santos ${ }^{2}$ \\ Gisele Maria de Godoi ${ }^{3}$ \\ Helena Maria Reis da Silva ${ }^{4}$ \\ Lucicleide da Costa e Silva ${ }^{5}$ \\ Vera Lúcia Catoto Dias ${ }^{6}$ \\ Anamaria da Silva Martin Gascón Oliveira ${ }^{7}$
}

Resumo: Este artigo visa complementar a proposta dos livros didáticos da rede municipal de Campos do Jordão. O tema abordado se refere à produção de um livro, que corrobora para uma melhor compreensão e manipulação do material concreto na construção da aprendizagem. O material estudado e produzido é referente ao conteúdo desenvolvido para a educação infantil, período escolar que é parte fundamental no desenvolvimento do educando, nessa idade a criança necessita de operações com material concreto, pois essa fase é base para uma educação alfabetizadora. As ações realizadas na fase inicial são de suma importância para todo o processo ensino-aprendizagem, pois as habilidades e os conceitos adquiridos serão utilizados durante todo o percurso do indivíduo.

Palavras-chave: Educação infantil; Material didático; Origem dos alimentos.

\footnotetext{
${ }^{1}$ Faculdade de Educação e Artes-Campus Platanus/UNIVAP, Brasil. E-mail: sarahs.sdsp@gmail.com.

2 Faculdade de Educação e Artes-Campus Platanus/UNIVAP, Brasil. E-mail: palomarosasantos@hotmail.com.

${ }^{3}$ Faculdade de Educação e Artes-Campus Platanus/UNIVAP, Brasil. E-mail: jaque_helena@hotmail.com.

${ }^{4}$ Faculdade de Educação e Artes-Campus Platanus/UNIVAP, Brasil. E-mail: helena.godoi.reis@gmail.com.

5 Faculdade de Educação e Artes-Campus Platanus/UNIVAP, Brasil. E-mail: slucicleide71@yahoo.com.

${ }^{6}$ Faculdade de Educação e Artes-Campus Platanus/UNIVAP, Brasil. E-mail: vcatoto@gmail.com.

${ }^{7}$ Faculdade de Educação e Artes-Campus Platanus/UNIVAP, Brasil. E-mail: gascon@univap.br.
} 